\title{
A Phenomenological Study of Facial Expression Animation
}

\author{
Robin J S Sloan \\ Institute of Arts Media and Computer Games \\ University of Abertay Dundee \\ r.sloan@abertay.ac.uk
}

\author{
Brian Robinson \\ Institute of Arts Media and Computer Games \\ University of Abertay Dundee \\ b.robinson@abertay.ac.uk
}

\author{
Ken Scott-Brown \\ Division of Psychology \\ University of Abertay Dundee \\ k.scott-brown@abertay.ac.uk
}

\author{
Fhionna Moore \\ Division of Psychology \\ University of Abertay Dundee \\ f.moore@abertay.ac.uk
}

\author{
Malcolm Cook \\ Division of Psychology \\ University of Abertay Dundee \\ m.cook@abertay.ac.uk
}

\begin{abstract}
This paper covers the findings of a qualitative study of facial animation, in which a cohort of student animators were tasked with producing spatiotemporally configured emotional expression animations. The timing of the upper and lower face regions within and between expressions such as happiness, sadness, and anger was explored by the animators, who sought to determine which configurations were the most and least effective in practice. The results showed that the student animators shared a degree of consensus when they discussed which configurations they found most authentic, and which configurations were the most clear. Configuration selection was dependent on the emotion or emotional transition being animated. These findings demonstrate that engagement with hand-key animators and practice-based research can generate results which would be of interest to the broader $\mathrm{HCl}$ community, in particular as regards the animation of interactive humanoid agents which exhibit believable changes in emotion.
\end{abstract}

Facial animation, emotional expression, hand-key animation, arts practice, animated agents

\section{INTRODUCTION AND BACKGROUND}

The development of embodied conversational agents (Cassell, 2001) for media interfaces is a well established field in $\mathrm{HCl}$ and computer graphics research. Interactive animated agents have been trialled with users, with findings demonstrating that agents can have a positive effect on user engagement (Bickmore et al., 2005) As well engaging users, a study of the effect of agent movement on observer perception has shown that animated agents are more effective at cueing attention than static images (Martinez et al., 2010). Research into the artificial intelligence of predictive agents (Murata et al., 2007) could substantially enhance the effectiveness of animated helper agents. In terms of application, a number of studies have sought to integrate interactive animated agents with virtual training and educational environments (Nunes et al., 2002; Woo, 2009). Effects of virtual learning intervention on child action have been recorded (Sapouna et al., 2010), supporting the notion that future animated agents will play a key role in the enhancement of $\mathrm{HCl}$ interfaces (Gratch et al., 2002).

The multimodality of animated agents - including speech, posture, and facial gestures - is a particular area of interest in current research (Beskow et al., 2007; Pelachaud, 2005). Agent animation is typically handled procedurally, drawing upon a library of animated sequences or transformations (Corradini et al., 2005). Specifically regarding facial animation, the work of Ekman and Friesen (1978) in developing FACS has informed research into computer animation of facial expressions (Arya et al., 2009; Deng and Noh, 2007). For the most part, facial animation for embodied conversational agents is based on morphing between accepted peak emotional expressions (Ekman and Friesen, 1975). More complex expressions which have subtle temporal 
dynamics have been proposed and applied in computer animation (Malatesta et al., 2006; Wehrle et al., 2000), while models of complex expression animation have been developed (Pelachaud, 2009). However, there has been little research into the potential for an ideal temporal configuration of dynamic facial expressions for agents. This is arguably due to the complexity of human expressions in nature.

One area which has received relatively little attention is the role of traditional animation practice in identifying ideal configurations for believable emotional expressions. In traditional animation, the principles of animation (Thomas and Johnston, 1981) and the artistic training students receive underpin the practice-based knowledge animators draw upon when making decisions. Traditional animators have a strong foundation in the observation of nature, but also hold tacit knowledge regarding the procedures for successfully imitating - rather than replicating - life. Previous research has shown that an arts-based approach to facial animation research can be adopted to generate detailed predictions regarding the clarity and authenticity of dynamic expressions (Sloan et al., 2009). Subsequent studies have demonstrated that the predictions of artistically produced configurations of facial movement - termed emotional expression choreography (EEC) - can be verified empirically through tests of observer perception (Sloan et al., 2010). As such, the rationale for the current research stems from the potential to interrogate the practice of animators in order to better understand believable - if not necessarily naturalistic - facial animation. By investigating how animators configure the subtle movement of emotional facial expressions, it is proposed that a better understanding of the movement of believable facial expressions can be revealed. This in turn could contribute to the procedural animation of perceptually valid embodied conversational agents for $\mathrm{HCl}$ interfaces.

\section{EMOTIONAL AVATARS PROJECT}

The current research forms part of an overall practice-based animation project entitled Emotional Avatars. The goal of the project is to lead investigation into facial animation dynamics through studio-based practice, substantiated by empirical and qualitative studies of animation perception and production. Central to Emotional Avatars is the concept of emotional expression choreography (EEC).

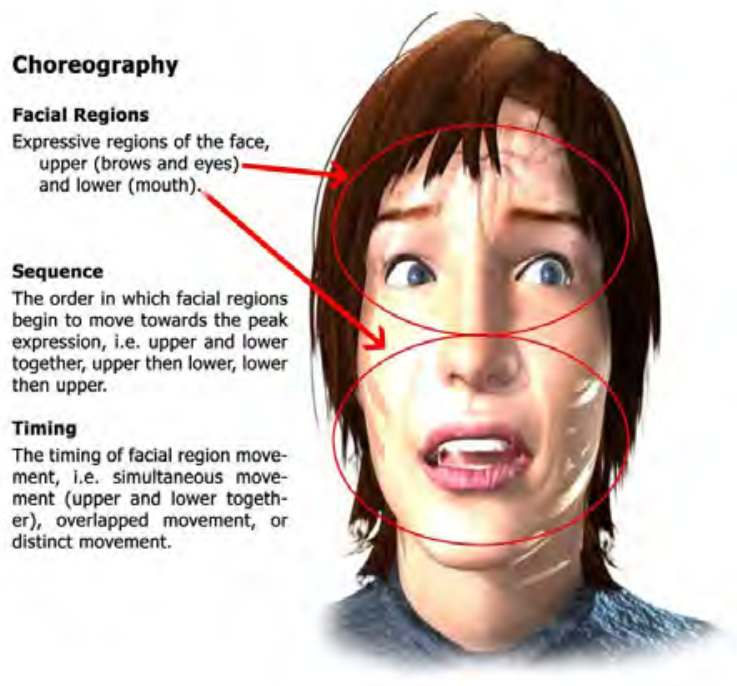

Figure 1: Emotional expression choreography

\subsection{Emotional expression choreography}

As discussed elsewhere (Sloan et al., 2010), the concept of EEC acts as a framework for hand-key animators tasked with producing nuanced emotional expression animation. Specifically, EEC relates to the sequence (Seq.) and timing (Tim.) of the upper and lower face regions during and between expressions. Figure 1 shows how the upper and lower face regions of an expression such as fear can be sequenced and timed by an animator. Using this framework, five configurations of subtle movement are possible; simultaneous movement of upper and lower regions (Seq.1), upper face leading with overlapped timing (Seq.2 Tim.A), upper face leading with distinct timing (Seq.2 Tim.B), lower face leading with overlapped timing (Seq.3 Tim.A), and lower face leading with distinct timing (Seq.3 Tim.B). A comparative example of how EEC configuration can affect expression appearance is shown in Figure 2, which depicts Seq. 2 Tim.B and Seq.3 Tim.B as applied to a transition between happiness and sadness.

Practice-based research using this framework demonstrated that experimentation with EEC could affect the animator's interpretation of expression authenticity, clarity, and context (Sloan et al., 2009, 2010). In addition, tests of observer perception demonstrated that manipulation of EEC can impact on audience interpretation of expression authenticity. However, previous studies sought only to explore the practice of a single animator, and to assess the measurable effects of EEC on observer perception. As identified earlier, traditional animation practice is largely dependent on artistic training and tacit knowledge. In order to explore 


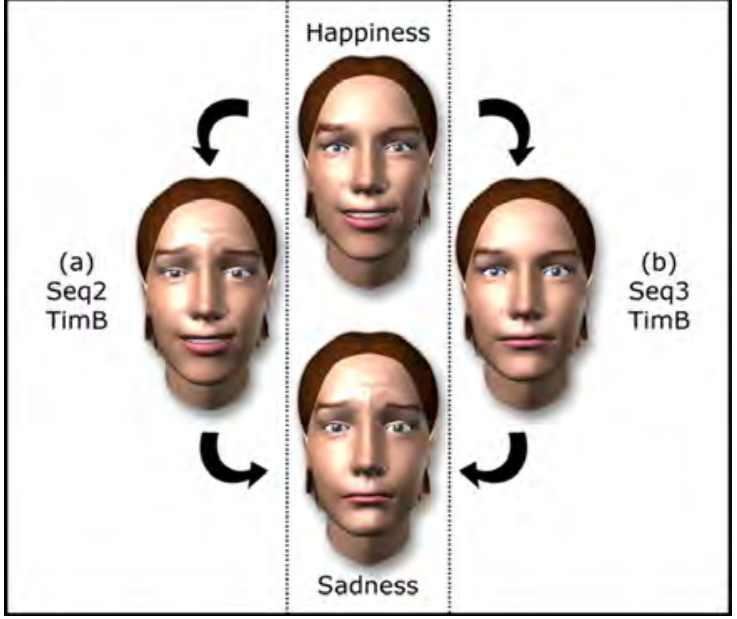

Figure 2: A transition of happiness into sadness animated using two choreographies

how animators as a group temporally configure facial animation, it was deemed necessary to conduct qualitative research into the experiences of these practitioners; in essence, to reveal insights into their practice-based understanding of what makes for good, emotional expression animation.

\section{METHODOLOGY}

In the current study, a group of student animators engaged with EEC in order to produce their own series of animated emotional expressions. The purpose of this study was to explore how participant animators interpreted the role of EEC and its potential effects, and to establish whether the participants held common beliefs regarding the application of particular choreographies to a series of emotional expressions. In order to collect and analyze qualitative data generated by the participants, the procedure of interpretative phenomenological analysis (IPA) discussed by Smith et al. (2009) was used as a framework. This form of thematic analysis was deemed most appropriate as the research was predominantly concerned with revealing the deeper, subjective perceptions of the involved participants after experiencing animation production. This method also explicitly accepts that the investigator can and should be part of the data collection process. Thus, any concerns that the investigator or interviewer might bias the process is removed.

\subsection{Participants}

In accordance with phenomenological methodology (Moustakas, 1994; Smith et al., 2009), participants were selected based on their experience with the phenomenon under investigation. For this study, the phenomenon was the experience of animating emotional facial expressions, in particular animating the expressions using the framework of EEC. As the EEC framework was unique at the time of the study, an existing sample was not available. To rectify this, steps were taken to manufacture a situation whereby potential participants could experience the phenomenon under fairly natural conditions. Two classes of computer arts and animation students were identified as participants. These were; one third year undergraduate class of approximately 29 , and one postgraduate class of approximately 16 . In order to expose these students to the concept of EEC, a series of lectures and tutorials were delivered which covered; the anatomy of the human head and face, the muscles and muscle groups activated during expressions (including an overview of FACS), the appearance of the universal expressions from artistic and scientific perspectives, the animation of facial expressions, and the proposed EEC framework. The group of approximately 45 students who attended the lectures and tutorials were invited to participate in an extended study of facial animation. From this group, seven went on to complete the project (four female, three male), Participants were aged between 21 and 30 . Five were enrolled in the third year of the undergraduate programme and two in the postgraduate programme. All seven participants had some experience working with specialist animation software (Maya 2008) prior to the start of the project, all had some artistic training and experience, and all had received official animation training as part of their studies. The participants worked on the project for 8-10 weeks individually and in their spare time. At the conclusion of the project, participants were paid $£ 20$ for their participation. In this paper, participants have been assigned pseudonyms (Luke, Helen, Patsy, Rory, Steven, Tricia and Wendy) in order to protect their anonymity.

\subsection{Procedure}

Participants were asked to create a range of facial animations using a provided facial rig (see Figure 3). Participants were asked to produce twelve facial animations, split into two sets of six. The first six animations were the universal expressions of emotion (happiness, sadness, anger, fear, disgust, and surprise), starting from a neutral face. The second set of animations were emotional expression transitions (surprise into happiness, happiness into sadness, sadness into anger, anger into fear, fear into disgust, and disgust into surprise). These expressions and transitions were previously assessed in experimental tests of observer perception (Sloan et al., 2010). For all scenarios, the participants were asked to experiment with the five EEC configurations. In order to record data during the animation production process, participants were requested to complete pre-animation questionnaires and post- 


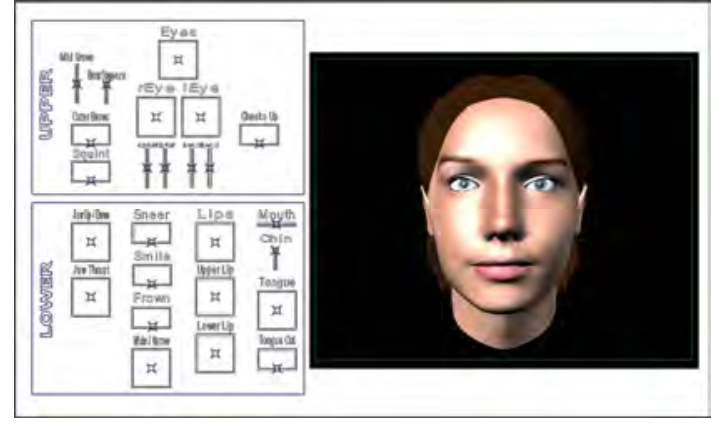

Figure 3: The facial animation rig used by participants

animation evaluations prior to submitting their work. Additionally, interviews were conducted to collect data on participant experience.

Due to the nature of exploratory qualitative research, no exact predictions were made regarding the outcome of this study. Nevertheless, it was expected that the participants would demonstrate a high level of reflective-practice during and after the generation of animation, and that, as a result, artistic interpretations of the role of EEC would be revealed. It was predicted that participants would offer detailed insights, developed through studio practice, which would demonstrate their interpretation of; the application of EEC, the effect of EEC on animation authenticity and clarity, and the wider context of EEC in animation. It was predicted that the current study would support previous research (Sloan et al., 2010) in demonstrating that studio practice can act as a catalyst for new knowledge regarding the movement of expressions, distinct from traditional experimental approaches.

\section{ANALYSIS AND RESULTS}

Following the IPA method for thematic analysis, diary documents and interview transcripts were firstly read in their entirety, before the documents were broken down into experiential statements. Descriptive, linguistic, and conceptual comments were noted alongside participant transcripts. These notes were then sent to participants for checking. Line by line analysis of participant transcripts informed the development of major themes and related meaning units. In particular, participants provided detailed interpretations of EEC and how it could affect; the authenticity of animation, the clarity of expressions, and the context and character of the expression. To help demonstrate where participants shared similar interpretations on the effects of EEC on particular expressions, coding for authenticity (including authentic and nonauthentic meaning units) has been summarised in Tables 1 and 2 within this paper. These results showed which choreographies the participant group interpreted as strongest and weakest for each emotional expression and transition. It should be noted, however, that these results are not meant to be generalised. They are instead presented as findings specific to the particular group of participants who were engaged with animation production using the EEC framework.

\subsection{Authenticity}

Firstly, participants were clearly able to extract their own artistic interpretation of how authentic EECs were when reflecting on practice. All seven participants made substantial reference to how authentic or inauthentic they believed an EEC was after experimenting with its application in practice. Beyond stating that animations appeared authentic or inauthentic, it was clear from the analysis that the participants considered the precise nature of the animations when reflecting on their work, including how natural or sincere choreographies appeared. For instance, expressional insincerity was often mentioned as a form of 'faking' which participants picked up on after producing an animation:

"If you were trying to show someone that was trying to be fake or was trying to be nervous, then different choreographies would work" (Helen)

Natural realism in the movement of features, dictated by EEC, was particularly prevalent in the interviews.

"I think some of them looked a bit odd ... your face wouldn't just move lower then top" (Tricia)

From this analysis, it was clear that the animators were simultaneously engaged in producing animation following the prescribed EEC guidelines, while casting a critical eye on the potential believability of the outcome. This ability to animate, assess realism and character sincerity (framed by artistic training and observation), and then refine the animations, showed that the participants were adept at describing their interpretation of authenticity. Ultimately, participant comments offered more insight into the effects of EEC on authenticity than, say, a simple measure of expression believability collected via questionnaire.

Of particular interest, it emerged that participants showed some agreement regarding the authenticity of the five choreographies in general. Six of the seven participants indicated a preference for upper face leading with overlapped timing (Seq.2 Tim.A) when discussing authenticity, while four participants indicated that lower face leading with distinct timing (Seq.3 Tim.B) was especially inauthentic: 
"I think quite natural is starting from upper face, and affecting the lower face. I think maybe this one includes all of them [all expressions] in it..." (Wendy)

"I felt that if the lower face peaked before the upper face, if the eyes didn't squint when the lower face peaked I felt that was quite fake. It didn't feel fluid, the eyes remained stationary" (Steven)

Through reflective-practice, the student animators were therefore aware of the how application of EEC affected their artistic interpretation of animation authenticity in terms of the realism of movement and the implied sincerity of expression. This theme will be revisited shortly in discussion of each of the animated expressions and transitions.

\subsection{Clarity}

Additionally, analysis of interview transcripts demonstrated that participants were interested in determining how EEC could affect expression clarity. Participants discussed how choice of EEC could impact on their interpretation of how clear or ambiguous a particular animation might be:

"You wouldn't be quite sure, some of them were looking more confused than disgusted, and it was like the lines were quite blurred, but then you'd do a different choreography, and all of a sudden the emotion would be quite clear, that it was disgust" (Helen)

"Some of them were very clear as to what emotion they would work for, others I think had barriers. You could use a couple depending on the scenario of it" (Tricia)

Again, expression identification is a common measure in facial animation experimentation, but here the animators were able to discuss in more detail the kind of issues EEC could raise as regards clarity.

\subsection{Context and character}

Analysis of the transcripts showed that all participants considered how choreography could be connected to the potential context or character of the animation. On context, participants broadly indicated that particular choreographies may be applicable to certain scenarios or a development of emotion. Broadly, context could be seen to be the defining factor, in that the application of EEC might be more dependent on the intended context than on the authenticity or clarity of the end result:

"So even though all my animations are simple, there is no context or story, but if context is applied to each scenario, then some of these other choreographies may be more useful. So all of them are right I think, it just depends on the context" (Tricia)

Expanding on this, precise scenarios for EEC were discussed, so that an EEC configuration might be selected by an animator dependent on what it is they want to achieve:

“...looking at how expressions are actually animated using different choreographies and stuff sort of shows how they'd be better off used in certain choreographies and certain situations. So you don't always use the same expression. It's the same expression but it's shown in a slightly different way depending on what you are doing" (Rory)

As regards character, participant comments in the interviews showed that engagement with choreography in animation production led to reflective insights on the nature of the character being animated. This included the potential thought process of the character, complex emotional states or feelings, and deliberate displays of emotion. Of particular interest was the participant's discussion of character thought process:

"Choreography 2 worked really well, because it really showed visually what was going on in the mind of the subject" (Steven)

"It looks more... thought provoking in most cases, because... it looks like you are thinking" (Wendy)

Evaluation of these comments was of particular interest, as it demonstrated that animation and reflection could offer clues as to the effect of EEC on aspects of the expressions which could not be explicitly measured (see Sloan et al., 2010 for a discussion of quantitative measures of identification and authenticity in expression animation).

\subsection{Intersubjective agreement}

As part of the process of completing the diaries, participants were required to make statements regarding their interpretation of EEC as applied to the range of expressions and transitions. In particular, participants declared which choreographies they felt were strongest and weakest. In general, it was apparent that participants opted to animate the expressions using either simultaneous movement (Seq.1) or upper face leading with overlapped timing (Seq.2 Tim.A). When stating which EEC they preferred for each expression, the majority discussed these two EECs for animations of; sadness, anger, fear, disgust, and surprise. Conversely, Seq. 3 Tim.B (leading with the lower face with distinct timing) was regarded as the weakest EEC in general, as participants highlighted it as being their least 
preferred choice when animating; sadness, anger, fear, and surprise. This trend was continued somewhat with the animation of expression transitions, with participants stating that Seq.1 or Seq.2 Tim.A were strongest for; happiness into sadness, sadness into anger, and anger into fear. Seq.3 Tim.B was outlined as the weakest EEC by the majority of participants for; sadness into anger, anger into fear, and disgust into surprise.

However, participant statements regarding the strongest and weakest EECs for each animation were not particularly detailed and offered little insight into the individual experience of each animator. More information was provided by participants elsewhere in the diaries and interviews regarding the three core themes of authenticity, clarity, and context and character. In the following sections, these three areas of interest shall be discussed in relation to the range of expressions and transitions animated.

\subsection{Animation of emotional expressions}

Firstly, participants discussed the animation of a happiness expression framed by EEC. Although participant interpretations of happiness EECs were varied, the authenticity of Seq.3 Tim.A, Seq.2 Tim.B and Seq.3 Tim.B was discussed by more than $50 \%$ of the animators. Leading with the lower face (Seq.3 Tim.A) was seen as being quite authentic, with participants describing it as being genuine, natural, and believable, while the other two EECs were broadly seen as being not authentic or faked. The effects on clarity were not discussed by many participants, but $4 / 7$ participants discussed context or character when evaluating Seq.1 and Seq.2 Tim.B. Simultaneous movement (Seq.1) was seen as being useful in certain contexts, in particular where the character was required to intentionally feign a smile (a social smile) or to laugh. Less agreement was apparent for the context of Seq.2 Tim.B, although the presence of a thought process was observed.

For sadness, it was evident that the participants strongly favoured leading from the upper face (Seq.2 Tim.A). Comments indicated that this choreography created an authentic and clear result - for example, it was regarded as the "most believable and easiest to read" (Steven), as a "genuine sadness" (Helen) and it was stated that it "looks and feels natural and believable" (Rory). There was strong emphasis on the tacit understanding of how easy it was to animate sadness using Seq.2 Tim.A - for example, that it "feels the most natural way to animate" (Tricia). Conversely, lower-face leading choreographies were seen as not authentic. In particular, Seq. 3 Tim.B was regarded as unnatural and insincere, with a range of comments indicating that it would only be useful in a context where sadness was "being forced" (Helen) or falsely displayed. Context was also discussed for Seq.2 Tim.B, which was identified as a potentially successful choreography but one which contained a higher degree of cognitive appraisal (or thought process).

As with sadness, the majority leaned towards Seq.2 Tim.A as an effective choreography for anger, and Seq.3 Tim.B as an ineffective choreography. The former was underlined as reliable, particularly so in that the resulting animation of anger was widely regarded as being unambiguous. In one example it was indicated that anticipation (a principle of animation) may be at play - "you can tell straight away the emotion about to be delivered" (Rory). The impact of the eyes and brows on the clarity of the expression made Seq. 2 Tim.A the most intuitive EEC for anger, and the participants stated that the outcome was believable. On the other hand, Seq.3 Tim.B was determined to be both inauthentic and ambiguous, with participants suggesting once more that this particular EEC made for "faked and forced" (Steven) and neither "natural" nor "believable" (Rory) animation. It was suggested that, not only would the animation be "hard to read" (Steven) but that the audience may interpret confusion (Patsy) or disgust (Tricia, Luke). Context and character were discussed for Seq.2 Tim.B, which was seen as being potentially appropriate for delayed or slowly developed feelings of anger, which contained more complexity and intensity.

The trend of favouring initial upper face movement over lower face movement continued with animations of fear. Seq.2 Tim.A was discussed as useful for animating what appeared to be a sincere, genuine expression of fear which was also natural in appearance and practical to produce. Simultaneous movement was regarded as the next best choice by the majority of participants, with Seq.1 also generating "genuine fear" (Helen) and "a more emotional response" (Patsy). The latter EEC was not perceived to be as intuitive, however, but it was identified that the speed of facial movement would be appropriate to the emotion. Seq.3 Tim.A and Tim.B, while not explicitly regarded as inauthentic, were seen to be emotionally ambiguous choreographies. Seq. 3 Tim.B in particular was highlighted as a confusing EEC, which was better suited to a "forced or faked emotion" (Steven). For disgust, simultaneous movement was the choreography which was discussed as the preferred choice by the majority of participants. However, there was less consistency across the more detailed comments for each of the EECs, and as such no one choreography stood out as the most or least authentic configuration. Similarly, the participants did now show agreement on the impact on expression clarity. This would 
suggest that the choreography of disgust is much more dependent on context as well as the subjective perspective of the animation practitioner. Context was discussed by many of the participants. Distinct timing choreographies were representative of a development of disgust, an insincere portrayal of disgust, or a mixture of disgust and anger. Overall, Seq.1 was regarded as the most practical choreography for this emotion.

Finally, animations of surprise were predominantly seen as effective using either Seq.1 or Seq.2 Tim.A, and ineffective using either Seq.3 Tim.A or Tim.B. Simultaneous movement was overwhelmingly seen as the most intuitive EEC for surprise - in that it "feels the most natural" (Helen) and was the choreography participants would "instinctively use" (Rory) - as well as being highly natural and sincere. Seq.2 Tim.A was regarded as a close second in terms of authenticity, with comments such as it being "also good to use" (Patsy) and "also a good choice" (Rory) indicating that Seq.1 was preferred by the majority. The choreographies that commenced with lower face movement were once again perceived as being unnatural and only appropriate for faked or exaggerated expressions of surprise.

Table 1 shows the coding frequency for participant discussion of authentic $(\checkmark)$ and inauthentic $(x)$ EECs as applied to the six universal expressions. Majority agreement in interpretation (more than or equal to $4 / 7$ participants) is shown here. The trend of favouring Seq.2 Tim.A followed by Seq.1 is clearly apparent, with the former being regarded by the majority as an authentic EEC for four emotions. Seq. 3 Tim.B was also regarded as inauthentic by the majority for four emotions. This trend was not universal, however - for instance, neither Seq.1 nor Seq.2 Tim.A were regarded as authentic for happiness or disgust, while Seq.3 Tim.A emerged as the most authentic EEC for happiness.

Table 1: Coding for EECs interpreted as most $(\checkmark)$ and least $(x)$ authentic by 7 participants

\begin{tabular}{|c|c|c|c|c|c|}
\hline \multirow{2}{*}{$\begin{array}{l}\text { Animated } \\
\text { Expression }\end{array}$} & \multirow[t]{2}{*}{ Seq.1 } & \multicolumn{2}{|l|}{ Seq.2 } & \multicolumn{2}{|l|}{ Seq. 3} \\
\hline & & Tim.A & Tim. $B$ & Tim.A & Tim.B \\
\hline Happiness & & & $x 4 / 7$ & $\checkmark 4 / 7$ & $x 4 / 7$ \\
\hline Sadness & & $\sqrt{6 / 7}$ & & $x 4 / 7$ & $\times 5 / 7$ \\
\hline Anger & & $\checkmark 5 / 7$ & & & $\times 5 / 7$ \\
\hline Fear & $\sqrt{4 / 7}$ & $\checkmark 5 / 7$ & & & \\
\hline Disgust & & & & & \\
\hline Surprise & $\sqrt{6 / 7}$ & $\checkmark 5 / 7$ & & $x 4 / 7$ & $x 4 / 7$ \\
\hline
\end{tabular}

\subsection{Animation of expression transitions}

The second set of participant animation and evaluation data concerned the production of emotional expression transitions. The animation of surprise into happiness was one of the few instances where participants indicated a preference for lower-leading choreographies. Both Seq.3 Tim.A and Tim.B were discussed as being authentic; as the "most genuine change in emotion (Helen) and "as the most natural" (Rory). Of the alternative EECs, it was Seq.2 Tim.B - upper face leading with distinct timing - that resulted in the most comments regarding inauthentic movement. The remaining choreographies sparked discussion on the potential context and character. However, there was little consistency between participant comments; Seq.1 was regarded as having either a specific context - as a faked expression (Helen) or reaction to a baby (Luke) - or no real context at all (Patsy, Wendy). Seq.2 Tim.A was seen as a choreography that would generate a more complex thought process, involving nervousness (Helen) or confusion (Tricia).

The only area of majority agreement in interpretation for the transition happiness into sadness was with the authenticity and artistically intuitive nature of simultaneous movement. Seq.1 was regarded as being "the most realistic" (Helen) and "most natural" (Rory) and was also the "most intuitive" (Luke) and "easiest to animate" (Tricia). Although there was little else said about the context of this particular EEC, the perceived reliability of Seq.1 over alternatives may suggest that more difficulty was found in animating effective transitions using more complex configurations. For instance, masking was identified as a specific context for Seq.2 Tim.B; the character could be "straining to hold back their sadness", and the result "would work very well in this context" (Steven). Two participants indicated that careful animation could create an effective transition using Seq.2 Tim.B; that if it was "done really well, it can look pretty good" (Luke) and that "if the timing is perfect it could work well" (Helen). Context was also discussed for Seq.3 Tim.A which was generally regarded as being a potentially useful choreography within certain contexts; for example "struggling to cope with the situation" (Steven) or "if the character ends up crying at the end" (Luke).

There was a high level of agreement between participants when choosing Seq.2 Tim.A as the strongest and Seq. 3 Tim.B as the weakest EECs for sadness into anger. All participants indicated that they felt the former was authentic - that it "felt the most realistic" (Steven) and was "not performed" (Wendy). In terms of context, this choreography was seen to convey a development from sadness to anger with a clear cognitive 
process. Conversely, Seq.3 Tim.B was regarded as inauthentic and ambiguous. For the most part, this perceived 'fakeness' was rooted in a belief that the animation appeared to be an insincere expression. The resulting transition was unclear, in that participants believed that it would "confuse the audience" (Patsy) or look like fear (Patsy) or disgust (Tricia). Simultaneous movement was also viewed as an inauthentic EEC by the majority of participants, who again stated that the quick change across the face resulted in a transition that appeared faked or acted.

Similar to the response to animating sadness into anger, participants showed a high level of agreement regarding Seq.2 Tim.A as authentic and Seq.3 Tim.B as inauthentic for the anger into fear transition. Across all of the major themes, it was only the authenticity of these two EECs where participants showed agreement, with none of the other themes being coded for more than $3 / 7$ of participant evaluation documents. Seq.2 Tim.A was regarded as an authentic transition as it was seen as a sincere reaction with natural, flowing movement. Individual participants also remarked that it was an artistically intuitive choice (Steven, Helen) and that the emotions were clearly staged (Patsy). As before, having the lower face peak before moving the upper face caused participants to interpret a faked or displayed emotion in their animation. The lack of initial upper face movement made later movement in the eyes appear excessively exaggerated (Helen) and generally created an animation which participants felt looked like faked emotions.

Analysis of participant evaluation documents for fear into disgust revealed that a majority of participants saw Seq.3 Tim.B as being potentially authentic. This was the only EEC which was regarded as authentic by the majority of participants, who suggested that it was a "unique transition between fear and disgust" (Steven) which appeared fairly authentic and believable. Within this choreography, participants commented that other thoughts or emotions could also be occurring, making it a more complex (yet still authentic) animation. Alternatively, leading with the upper face with distinct timing (Seq.2 Tim.B) was interpreted as an inauthentic EEC as it "felt slightly unnatural" (Steven), "proved unnatural to watch" (Rory), and even appeared "very fake" (Helen). A context which called for a more deliberate development of emotion over time was identified as a potential scenario for this EEC.

Lastly, participants found that Seq. 1 and Seq.2 Tim.A were the most intuitive choreographies for disgust into surprise, in line with the previous study of neutral into surprise. Analysis of participant comments showed that most participants felt that
Table 2: Coding for EECs interpreted as most $(\curvearrowleft$ and least $(x)$ authentic by 7 participants

\begin{tabular}{|c|c|c|c|c|c|}
\hline \multirow{2}{*}{$\begin{array}{l}\text { Animated } \\
\text { Transition }\end{array}$} & \multirow[t]{2}{*}{ Seq.1 } & \multicolumn{2}{|l|}{ Seq.2 } & \multicolumn{2}{|l|}{ Seq.3 } \\
\hline & & Tim.A & Tim.B & Tim.A & Tim.B \\
\hline $\begin{array}{l}\text { Surprise } \\
\text { into } \\
\text { Happiness }\end{array}$ & & & $x \quad 4 / 7$ & $\sqrt{6 / 7}$ & $\checkmark 4 / 7$ \\
\hline $\begin{array}{l}\text { Happiness } \\
\text { into } \\
\text { Sadness }\end{array}$ & $\checkmark 4 / 7$ & & & & \\
\hline $\begin{array}{l}\text { Sadness } \\
\text { into Anger }\end{array}$ & $x \quad 4 / 7$ & $\checkmark 7 / 7$ & & & $x \quad 4 / 7$ \\
\hline $\begin{array}{l}\text { Anger into } \\
\text { Fear }\end{array}$ & & $\sqrt{ } 5 / 7$ & & $x \quad 5 / 7$ & \\
\hline $\begin{array}{l}\text { Fear into } \\
\text { Disgust }\end{array}$ & & & $x \quad 4 / 7$ & & $\checkmark 5 / 7$ \\
\hline $\begin{array}{l}\text { Disgust } \\
\text { into } \\
\text { Surprise }\end{array}$ & $x \quad 4 / 7$ & $\checkmark 4 / 7$ & & & \\
\hline
\end{tabular}

Seq.2 Tim.A was authentic, as it was a "reliable representation of the transition coupled with believable timing" (Steven) and "looked the most genuine" (Helen). An unexpected finding here was that, despite being perceived as intuitive, most participants actually found Seq.1 to be an inauthentic EEC for disgust into surprise. While three participants interpreted it as being fairly authentic, the remaining participants suggested that it "did not look natural" (Rory) and that it "looks like a meaningless transition" (Wendy).

Table 2 shows the coding frequency for participant discussion of authentic $(\checkmark)$ and inauthentic $(x)$ EECs as applied to the six expression transitions. As before, majority agreement in interpretation (more than or equal to $4 / 7$ participants) is highlighted. There was less consistency in EEC selection for the transitions, with no EEC identified as the most or least authentic for more than three of the animations. Seq.3 Tim.B was deemed authentic by the majority in two instances; surprise into happiness, and fear into disgust. Meanwhile, Seq.2 Tim.A was regarded as authentic for; sadness into anger, anger into fear, and disgust into surprise. These results are arguably more interesting than those from the first study of universal expression animations, in that the perceived authenticity of an EEC was more clearly dependent on the transition being animated.

\section{DISCUSSION}

The reported study provides some evidence that the engagement with EEC can trigger a degree of intersubjective agreement between practitioners when applied in the studio. In this case, the experiences of seven student animators were 
considered. Firstly, the selection and application of thematic analysis following the IPA procedure facilitated the emergence of three core themes related to the application of EEC; authenticity, clarity, and context and character. These themes related to previous practice-based (Sloan et al., 2009) and empirical (Sloan et al., 2010) findings, in particular as regards the measurement of expression authenticity and observer identification of emotion. However, the current study reveals a broader range of meaning units under the core themes, for example in relation to the sincerity of an authentic expression. Additionally, the analysis of participant interpretation of the effects of choreography on context and character painted a more detailed picture of EEC, one which was not possible to establish through previous experimental research. In consequence, tentative recommendations can be made regarding the precise application of temporally configured facial animation, beyond simple notions of clarity and perceived authenticity.

It was clear that majority agreement in interpretation was reached in most cases when animators declared which choreographies they felt were most and least authentic (see Tables 1 and 2). In general, it appeared as though Seq.2 Tim.A was regarded as the best EEC after analysis of participant interpretation of authenticity, while Seq.3 Tim.B was regarded as the weakest EEC for most expressions and transitions. This was in line with the findings of the previous research into the effect of EEC on audiences (Sloan et al., 2010), which revealed that leading with the upper face with overlapped timing was most acceptable, while leading with the lower face with distinct timing was more difficult to apply. However, when each of the twelve expressions and transitions were examined individually, it was evident that the nature of the emotion being expressed modulated participant interpretation. While Seq.2 Tim.A was generally strong, participants were more drawn to the lower face choreographies for animations of; happiness, surprise into happiness, and fear into disgust. Furthermore, simultaneous movement (Seq.1) was preferred by the majority for; fear, surprise, and happiness into sadness. Although there was fluctuation in individual interpretation of context and character, in most cases one or two EECs were identified by the majority as the most or least authentic. There was less agreement regarding the impact on expression clarity. Nevertheless, authenticity was perhaps the more important consideration, as it related directly to the idea that EEC could manipulate the believability of animated expressions.

The findings of this study would therefore indicate that - from the perspective of student animators after practice-based experimentation - dynamic expressions of animated agents work best when the upper face leads with overlapped timing. Generally, animated expressions do not work particularly well when the lower face leads with distinct timing. However, this rule of thumb cannot be applied broadly across all dynamic facial expressions, and in certain circumstances alternative temporal configurations are more appropriate. Although participant data would suggest that the clarity of an expression is rarely compromised through manipulation of EEC, the findings show that selection of a particular EEC could positively or negatively affect the authenticity of the expression or transition. In particular, participants drew attention to the fact that selection of the wrong EEC could create an expression which might be perceived as masked, faked, or insincere. Overall, the results of this study demonstrate that a straight blend between two expressions (Seq.1) is rarely the best option when looking to generate a believable change of expression. Artistically at least, it is necessary to consider the subtlety of movement within and between emotional expressions when generating facial animation for embodied agents.

\section{FURTHER RESEARCH}

The findings discussed in this paper relate to the artistic design process for effective animated agents, specifically the timing of movement within and between expressions. Following on from this, more work is needed in order to develop a full taxonomical account of artistically tested expression movement. This future work should target more complex changes in expression (such as mixed emotion, cognitive process, and other non-emotional expressions). Additionally, an empirical study of the effects of configured expression animation on observer perception and attention should be conducted to evaluate how effective these animations are within user interfaces.

\section{REFERENCES}

Arya, A., DiPaola, S. and Parush, A. (2009) Perceptually valid facial expressions for characterbased applications. International Journal of Computer Games Technology, Article ID 462315,

Beskow, J., Granström, B. and House, D. (2007) Analysis and synthesis of multimodal verbal and non-verbal interaction for animated interface agents. Proceedings of the 2007 COST action international conference on verbal and nonverbal communication behaviours, pp. 250-263. SpringerVerlag, Berlin.

Bickmore, T. W., Caruso, L. and Clough-Gorr, K. (2005) Acceptance and usability of a relational agent interface by urban older adults. In $\mathrm{CHI}$ ' 05 
extended abstracts on Human factors in computing systems (CHI '05), pp. 1212-1215. ACM, New York.

Cassell, J. (2001) Embodied conversational agents: representation and intelligence in user interface. Al Magazine, 22, 4, 67-83.

Corradini, A., Mehta, M., Bernsen, N. O. and Charfuelan, M. (2005) Animating an interactive conversational character for an educational game system. In Proceedings of the 10th international conference on Intelligent user interfaces (IUI '05), pp. 183-190. ACM, New York.

Deng, Z. and Noh, J. (2007) Computer facial animation: a survey. In Deng, Z. and Neumann, U. (eds), Data-Driven 3D Facial Animation. Springer, London.

Ekman, P. and Friesen, W. V. (1975) Unmasking the face. Prentice Hall, New Jersey.

Ekman, P. and Friesen, W. V. (1978) Facial action coding system: a technique for the measurement of facial movement. Consulting Psychologists Press, Palo Alto.

Gratch, J., Rickel, J., Andre, E., Cassell, J., Petajan, E. and Badler, N. (2002) Creating interactive virtual humans: some assembly required. IEEE Intelligent Systems, 17, 4, pp. 5463.

Malatesta, L., Raouzaiou, A. and Kollias, S. (2006) MPEG-4 facial expression synthesis based on appraisal theory. In Maglogiannis, I., K. Karpouzis, K. and Bramer, M. (eds). Artificial Intelligence Applications and Innovations, pp. 378-384. Springer, Boston.

Martinez, S., Sloan, R. J. S., Szymkowiak, A. and Scott-Brown, K. (2010) Using virtual agents to cue observer attention: assessment of the impact of agent animation. CONTENT 2010, Lisbon, Portugal, 21-26 November.

Moustakas, C. (1994) Phenomenological research methods. SAGE Publications, Thousand Oaks.

Murata, K., Enomoto, M., Arimoto, Y. and Nakano, Y. (2007) When should animated agents give additional instructions to users? Monitoring user's understanding in multimodal dialogues. Control, Automation and Systems 2007 (ICCAS 07), Seoul, Republic of Korea, 17-20 October.

Nunes, M., Dihl, L., Fraga, L., Woszezenki, C., Oliveira, L., Francisco, D., Machado, G., Nogueira,C. and Notargiacomo, M. (2002) Animated pedagogical agent in the intelligent virtual teaching environment. Interactive Educational Multimedia, 4, 53-60.

Pelachaud, C. (2005) Multimodal expressive embodied conversational agents. Proceedings of the 13th annual ACM international conference on Multimedia (MULTIMEDIA '05), pp. 683-689. ACM, New York.

Pelachaud, C. (2009) Modelling multimodal expression of emotion in a virtual agent. Philosophical Transactions of the Royal Society $B$, 364, pp. 3539-3548.
Sapouna, M. et al. (2010) Virtual learning intervention to reduce bullying victimization in primary school: a controlled trial. Journal of Child Psychology and Psychiatry, 51, 1, pp. 104-112.

Sloan, R., Robinson, B. and Cook, M. (2009) Investigating facial animation production through artistic inquiry. In Computation World 09, Athens, Greece, 15-20 November, pp. 675-681.

Sloan, R.J.S., Robinson, B., Scott-Brown, K., Moore, F. and Cook, M. (2010) Choreographing emotional facial expressions. Computer Animation and Virtual Worlds, 21, 3-4, pp. 203-213.

Smith, J.A., Flowers, P. and Larkin, M. (2009) Interpretive phenomenological analysis: theory, method and research. SAGE Publications, London. Thomas, F. and Johnston, O. (1981) The illusion of life: disney animation. Disney Editions, New York. Wehrle, T., Kaiser, S., Schmidt, S. and Scherer, K.R. (2000) Studying the dynamics of emotional expression using synthesized facial muscle movements. Journal of Personality and Social Psychology, 78, 1, pp. 105-119.

Woo, H.L. (2009) Designing multimedia learning environments using animated pedagogical agents: factors and issues. Journal of Computer Assisted Learning. 25, 3, pp. 203-218. 